\title{
Skin Detection Using Color Distance Measurement And Thresholding
}

\author{
Dolly Indra, Lilis Nur Hayati
}

\begin{abstract}
Segmentation is a process to extract the objects in an image. In this study, segmentation process is used to perform skin detection which aims to detect skin pixels and areas in an image. Pixel-based measures is used in the similarity measurement model in image area segmentation to detect skin and non-skin areas in 3 color spaces i.e. RGB with Euclidean distance, $\mathrm{HSV}$ with Dcyl distance, $\mathrm{HCL}$ with $\mathrm{DHCL}$ distance and the thresholding value as the limit of the similarity between two colors. The results of this study indicate that color space segmentation process using thresholding value (Th $\leqq 80$ and Th $\leqq 100)$ for HSV and HCL provide superior segmentations.
\end{abstract}

Keywords: Segmentation; RGB; HSV; HCL; Threshold; Euclidean Distance; Dcyl distance; DHCL distance.

\section{INTRODUCTION}

Segmentation is one of the most important parts in image processing used for image analysis and pattern recognition [1], [2]. The process aims to represent the area of an image into a number of segments which are more meaningful and easier to analyze [3].

Image color segmentation is a segmentation process on image based on the similarity of color. The main process is to determine pixels included in a segment that has different color maps and require a tool to measure the difference between the two colors. Pixel-based segmentation is a segmentation based on color distance measurement between two neighboring pixels [4]. Thresholding is one of the most important segmentation techniques used as a two-color similarity limit [5]-[7].

The purpose of this study is to extract object of interest from other objects in order to simplify the next analysis. The segmentation uses color distance measurement and threshold value (Th) to determine the similarity of the color of skin to the color of each pixel in the image [8], [9]. This matter is performed in order to distinguish between the skin and non-skin areas. The color space used in this study are the RGB, HSV, and HCL.

\section{EXPERIMENTAL DETAILS}

In this study, we use the similarity measurement model i.e. pixel based segmentation and thresholding. Pixel-based color image segmentation is semi-automated, an operator intervention is required to determine the color of the ROI (Region of Interest) of the image. The research which have been performed state that pixel-based has a good accuracy [10], [11].

The proposed system design on skin detection i.e. i). Read the Image, ii). Determine the ROI, iii). Determine Threshold Value, iii). Conversion to RGB, HSV, and HCL, iv). Calculate the distance RGB, HSV, and HCL, v). Display the result of RGB, HSV, and HCL color segmentation.

The input image is a hand image in portable network graphic (png) format. We performed a separation between skin and non-skin area. ROI is the type of color in the area of an image desired by the user to be segmented. Thresholding is used to group the neighboring pixels and has smaller or equal color distance to the threshold value as the similarity boundary between two colors. Thresholding is a simple but powerful approach in segmentation process. In conversion step, we convert the image from RGB to HSV and RGB to HCL. The RGB color space consists of three color components: Red, Green, and Blue. Each component has 8-bit value [12]. The HSV color space consists of 3 components i.e. hue, saturation, and value [13]. The color model is also known as the hexacone model which is the vertical axis center represents the intensity. Conversion from RGB to HSV is performed in the (1).

$$
\begin{gathered}
V=\operatorname{Max}, H=\left\{\begin{array}{c}
\text { Undefined, if Max }=\text { Min } \\
\left(\frac{G-B}{\operatorname{Max}-\operatorname{Min}}\right) * A, \text { if } \operatorname{Max}=R \\
\left(\frac{B-R}{\operatorname{Max}-\operatorname{Min}}+2\right) * A, \text { if } \operatorname{Max}=G \\
\left(\frac{R-G}{\operatorname{Max}-\operatorname{Min}}+4\right) A, \text { if } \operatorname{Max}=B
\end{array}, \quad S=\right. \\
\left\{\begin{array}{c}
0, \text { if Max }=\text { Min } \\
\text { Max -Min , if another }
\end{array}\right.
\end{gathered}
$$

As pointed in (1) the $\operatorname{Max}=\max (\mathrm{R}, \mathrm{G}, \mathrm{B}), \min =$ $\min (\mathrm{R}, \mathrm{G}, \mathrm{B})$ and $A=\pi / 3$ when $\mathrm{H}$ is expressed in radians, or $\mathrm{A}=60^{\circ}$ when $\mathrm{H}$ is expressed in degrees. The component value of $H$ in radians is vary, from $(-\pi) / 3$ to $(+5 \pi) / 3$ or in degrees from $60^{\circ}$ to $300^{\circ}$ while the saturation value $(\mathrm{S})$ and value or luminance value (V) from 0 to 255 . The HCL color space consists of 3 components i.e Hue, Chroma, and Luminance [14]. As shown in (2), it is used to convert from RGB color space to HCL color space.

$$
\begin{array}{r}
L=\frac{Q * \operatorname{Max}(R, G, B)+(Q-1) * \operatorname{Min}(R, G, B)}{2}, \\
C=\frac{Q *(|R-G|+|G-B|+|B-R|}{3}, H=\operatorname{atan}\left(\frac{G-B}{R-G}\right)
\end{array}
$$

\footnotetext{
Revised Manuscript Received on April 19, 2019.

Dolly Indra, Faculty of Computer Science, Universitas Muslim Indonesia, Makassar, Indonesia. (Email: dolly.indra@umi.ac.id)

Lilis Nur Hayati, Faculty of Computer Science, Universitas Muslim Indonesia, Makassar, Indonesia.
} 
RGB color space using Euclidean distance shown in the equation (3). As pointed in (3), R1, G1, B1 and R2, G2, B2 are components of red, green, blue from the first pixel and the second pixel.

$$
\Delta E=\sqrt{\left(R_{1}-R_{2}\right)^{2}+\left(G_{1}-G_{2}\right)^{2}+\left(B_{1}-B_{2}\right)^{2}}
$$

HSV color space using the Dcyl formula is shown in the (4), and the $\mathrm{HCl}$ color space using the $\mathrm{D}_{\mathrm{HCL}}$ formula is shown in the (5)

$$
\begin{aligned}
& D_{c y l}= \\
& \sqrt{\left(L_{1}-L_{2}\right)^{2}+\left(C_{1}\right)^{2}+\left(C_{2}\right)^{2}-2 C_{1} C_{2} \cos \left(H_{1}-H_{2}\right)} \\
& D_{H C L}= \\
& \sqrt{A_{L}\left(L_{1}-L_{2}\right)^{2}+A_{C H}\left(C_{1}^{2}+C_{2}^{2}-2 C_{1} C_{2} \cos \left(H_{1}-H_{2}\right)\right)}
\end{aligned}
$$

Where $\mathrm{S}$ in the color space of HSV is identical to Chroma (C) in the HCL, whereas Value (V) in the HSV color space is identical to Luminance in $\mathrm{HCL}, \mathrm{A}_{\mathrm{L}}=1.4456$ dan $\mathrm{A}_{\mathrm{CH}}$ $=|\Delta H|+0.16^{0}$, when $\Delta H$ is expressed in degrees or $\mathrm{A}_{\mathrm{CH}}=$ $|\Delta H|+\left(0.16^{0} *{ }^{p i} / 180^{\circ}\right)$ when $\mathrm{H}$ is expressed in radians.

The algorithm for extracting skin or non-skin areas as ROI is as follows: (1) Input image. (2) Determine the pixel color area of ROI as the pixel color for segmentation. (3) Determine color distance threshold value. (4) Conversion of RGB to HSV and Conversion of RGB to HCL. (5) For each pixel in the image, calculate the color distance against the reference color, if the color distance is smaller than the threshold value then it is part of the segment area otherwise, the pixel is not part of the segment area.

\section{RESULT AND DISCUSSION}

In this study, we used pixel-based measures with the semi-automated that requires the operator or user to determine the ROI. Pixels of RGB are used as reference colors for color distance measurements in RGB space. Conversion of pixel to HSV will be used as the reference color for the color distance measuring in the HSV, while conversion of pixel to the HCL will be used as the reference color for the color distance measurement in the HCL. The threshold value (Th) is used as a two-color similarity limit of $40,60,80$, and 100 . In figure 1 , the blue point is the color pixel selected as the reference color for extracting the area according to the color.
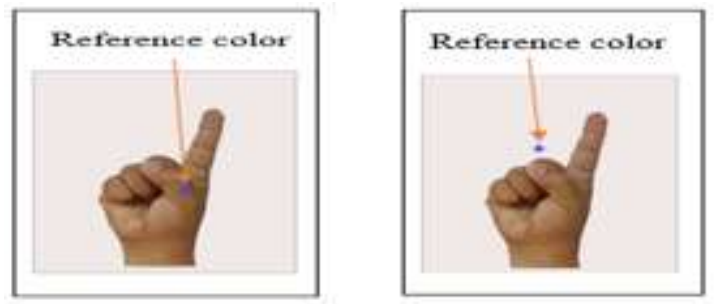

Fig. 1: Color ROI

Figure 2(a) is the result of segmentation by using Euclidean formula with RGB color space. Figure 2(b) is the result of segmentation by using the Dcyl formula with the color space of HSV and image 2(c) is the result of segmentation by using the DHCL formula with the HCL color space.

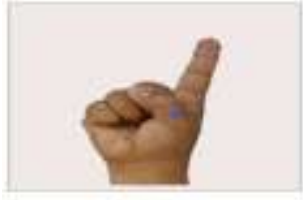

(a)

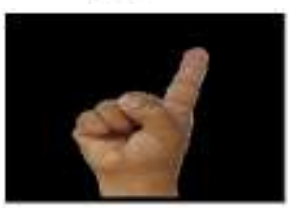

(c)

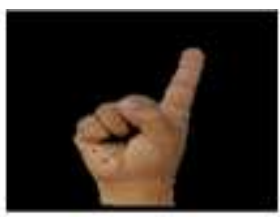

(b)

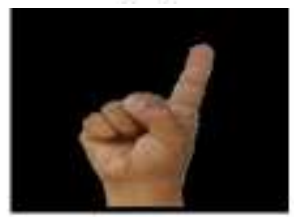

(d)
IMAGE

Fig. 2: Segmentation process RGB $(T h \leqq 40) \quad$ HSV $(T h \leqq 40)$

HCL $(T h \leqq 40)$
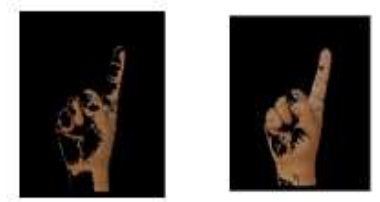

RGB $(T h \leqq 60)$

$\mathrm{HSV}(T h \leqq 60)$
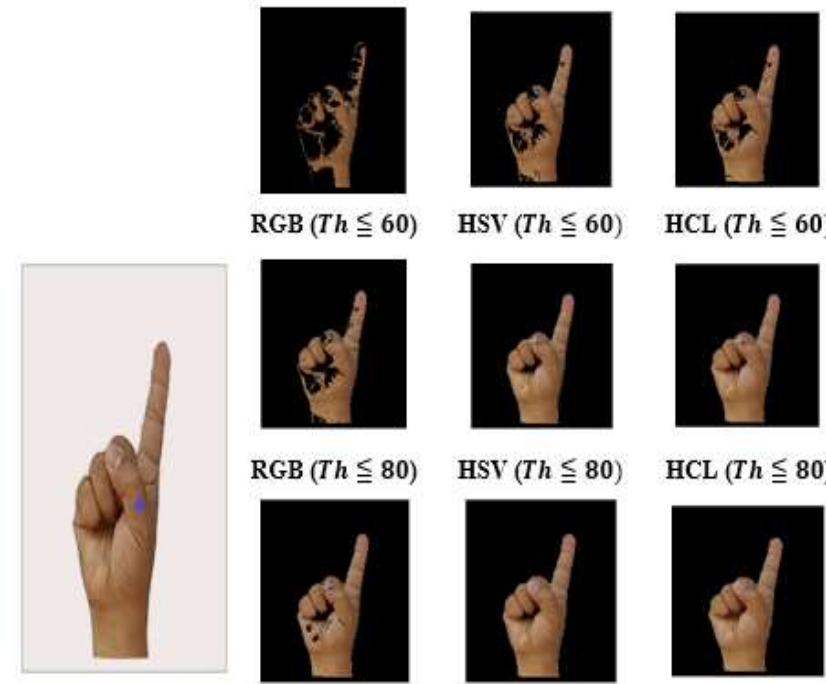

HCL $(T h \leqq 60)$

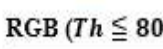

HSV $(T h \leqq 80)$
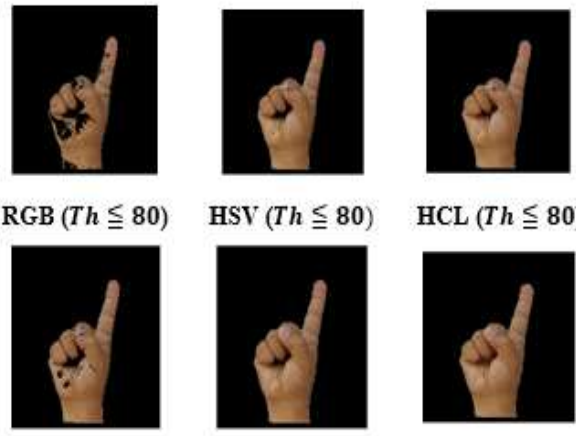

HCL $(T h \leqq 80)$

$\operatorname{RGB}(T h \leqq 100)$

HSV $(T h \leqq 100)$
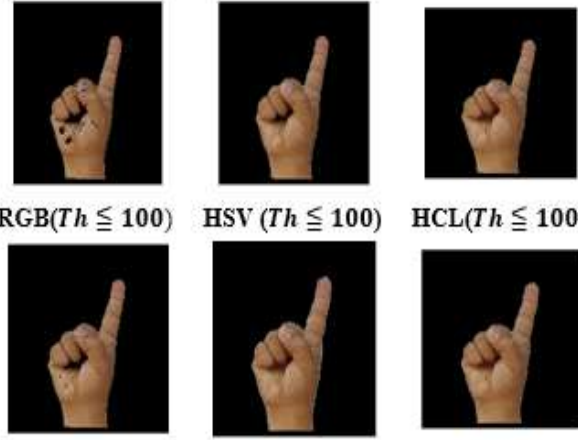

$\mathrm{HCL}(T h \leqq 100)$

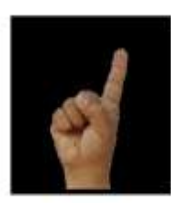

Fig. 3: Segmentation of skin area

\section{CONCLUSION}

The methods and algorithms we have used for color segmentation in this study were able to separate the skin and non-skin areas in the image by using the reference color as ROI by adjusting the threshold value according to the similarity precision level. The future work is the development of pixel-based measurement algorithms and performing extraction in handshape features.

\section{REFERENCES}

1. H. J. Vala and A. Baxi, "A review on Otsu image segmentation algorithm," Int. J. Adv. Res. Comput. Eng. Technol., vol. 2, no. 2, pp. 387-389, 2013. 
2. N. Kaur, V. K. Banga, and A. Kaur, "Image Segmentation Based On Color," Int. J. Res. Eng. Technol., vol. 2, no. 11, 2013.

3. A. De and C. Guo, "An image segmentation method based on the fusion of vector quantization and edge detection with applications to medical image processing,” Int. J. Mach. Learn. Cybern., vol. 5, no. 4, pp. 543-551, 2014.

4. M. Shebl, M.-T. El, T. Fathy, Y. Mogahed, and M.-H. El, "Distance measurement using proximity sensor in pedestrian and bicycle navigation," Int. J. Eng. Technol., vol. 7, no. 4, pp. 4266-4270, 2018.

5. N. Senthilkumaran and S. Vaithegi, "Image segmentation by using thresholding techniques for medical images," Comput. Sci. Eng. An Int. J., vol. 6, no. 1, pp. 1-13, 2016.

6. M. Chandrakala and P. D. Devi, "Threshold Based Segmentation Using Block Processing," Int. J. Innov. Res. Comput. Commun. Eng., vol. 4, no. 1, pp. 821-826, 2016.

7. U. C. De, M. Das, and D. Mishra, "Threshold based brain tumor image segmentation,” Int. J. Eng. Technol., vol. 7, no. 3, pp. 1801-1803, 2018.

8. R. Harrabi and E. B. Braiek, "Color Image Segmentation by Multilevel Thresholding using a Two Stage Optimization Approach and Fusion,” Int. J. Eng. Innov. Technol., vol. 3, no. 11, pp. 13-20, 2014.

9. H. K. Singh, S. K. Tomar, and P. K. Maurya, "Thresholding Techniques applied for Segmentation of RGB and multispectral images," Proc. Publ. by Int. J. Comput. Appl. SSN, pp. 24-27, 2012.

10. H. E. Adam, E. Csaplovics, and M. E. Elhaja, "A comparison of pixel-based and object-based approaches for land use land cover classification in semi-arid areas, Sudan," 2016.

11. B. Salehi, Y. Zhang, and M. Zhong, "Combination of object-based and pixel-based image analysis for classification of vhr imagery over urban areas," in Proceedings of ASPRS 2011 Annual Conference, 2011.

12. P. Ganesan, P. Chakravarty, and S. Verma, "Segmentation of natural color images in HSI color space based on FCM clustering," Int. J. Adv. Res. Comput. Eng. Technol., vol. 3, no. 3, 2014.

13. Z. Liu, W. Chen, Y. Zou, and C. Hu, "Regions of interest extraction based on HSV color space," in IEEE 10th International Conference on Industrial Informatics, 2012, pp. 481-485.

14. S.Kolkur, D. Kalbande, P.Shimpi, C.Bapat, and J. Jatakia, "Human Skin Detection Using RGB, HSV and YCbCr Color Models," in ICCASP/ICMMD-2016. Advances inIntelligent Systems Research, 2017, vol. 137, pp. 324-332. 